\title{
Prevalência e desfecho da tuberculose no Estado de Goiás
}

\author{
Prevalence and outcome of tuberculosis in the State of Goiás \\ Prevalencia y resultados de la tuberculosis em el Estado de Goiás
}

Ana Flávia Ribeiro Vilela

ORCID: https://orcid.org/0000-0002-5462-7867

Universidade de Rio Verde, Brasil

E-mail: anaflaviavilela08@gmail.com

Gabriela Melo

ORCID: https://orcid.org/0000-0001-8479-0318

Universidade de Rio Verde, Brasil

E-mail: gabrielamelop3@gmail.com

Flávia Caroline Silva Neves

ORCID: https://orcid.org/0000-0002-6948-5806

Universidade de Rio Verde, Brasil

E-mail: flaviaroline@gmail.com

Gabriella Almeida Silva dos Reis

ORCID: https://orcid.org/0000-0002-7609-2330

Universidade de Rio Verde, Brasil

E-mail: gabriellareisa@outlook.com

Gabriele Martins Lima

ORCID: https://orcid.org/0000-0002-6736-8630 Universidade de Rio Verde, Brasil

E-mail: gabriele@gmail.com

Gabriel de Castro Monteiro

ORCID: https://orcid.org/0000-0002-2986-9303

Universidade de Rio Verde, Brasil

E-mail: gabrielcasteiro@gmail.com

Italo Marcucci

ORCID: https://orcid.org/0000-0002-1851-7767

Universidade de Rio Verde, Brasil

E-mail: italomarcucci@hotmail.com

João Lucas Rocha Silva

ORCID: https://orcid.org/0000-0003-3469-156X

Universidade de Rio Verde, Brasil

E-mail: joaolucasrocha.2014@gmail.com

Pedro Henrique Santos Lima

ORCID: https://orcid.org/0000-0002-5748-0311 Universidade de Rio Verde, Brasil

E-mail: pedrohs164@gmail.com

Weberton Dorásio Sobrinho

ORCID: https://orcid.org/0000-0003-0619-7214

Universidade de Rio Verde, Brasil

E-mail: dorasioweberton@gmail.com

Danyelly Rodrigues Azevedo

ORCID: https://orcid.org/0000-0003-2001-6379

Universidade de Rio Verde, Brasil

E-mail: danyelly.rodrigues@unirv.edu.br

Raiane Antunes Sampaio

ORCID: https://orcid.org/0000-0003-1011-7088

Universidade de Rio Verde, Brasil

E-mail: raiane.sampaio@unirv.edu.br

\section{Resumo}

A tuberculose é uma doença infecciosa causada pelo bacilo Mycobacterium tuberculosis. É uma doença evitável e curável, mas apesar disso, continua a ser uma séria ameaça à saúde pública global, sendo a segunda causa de morte entre as doenças transmissíveis. O estudo tem como objetivo identificar a prevalência e a adesão ao tratamento relacionado aos desfechos cura, abandono e óbito por tuberculose no estado de Goiás nos anos de 2016 a 2020 . Tratase de um estudo epidemiológico transversal de aspecto quantitativo por meio do DATASUS/TABNET, utilizando as variáveis, ano de notificação, ano de diagnóstico, ano início de tratamento, sexo e situação encerrada envolvendo a cura, abandono e óbito por tuberculose e foi. Nesse sentido, os maiores números de notificações ocorreram no ano de 2018 e os menores, no ano de 2020. Em relação à cura, abandono de tratamento e óbito por tuberculose no período 
indicado, os maiores percentuais foram: 79,35\% em 2016, 22,74\% em 2020 e 10,83\% em 2020, respectivamente. Em relação ao sexo, a maior porcentagem foi do sexo masculino, sendo $74,27 \%$, que foram associados a uma menor taxa de cura, maior taxa de abandono do tratamento e a uma maior taxa de óbitos por tuberculose, quando comparados com o sexo feminino, presente em $25,72 \%$ dos casos totais. Portanto, os resultados demonstram uma porcentagem significativa dos casos com predominância no gênero masculino relacionado à menor taxa de cura, maior taxa de abandono ao tratamento e maior taxa de óbitos.

Palavras-chave: Tuberculose; Cura; Óbitos; Sexo masculino.

\begin{abstract}
Tuberculosis is an infectious disease caused by the bacillus Mycobacterium tuberculosis. It is a preventable and curable disease, but despite this, it remains a serious threat to global public health, being the second cause of death among communicable diseases. The study aims to identify the prevalence and adherence to treatment related to the outcomes cure, abandonment and death from tuberculosis in the state of Goiás in the years 2016 to 2020 . This is a cross-sectional epidemiological study of quantitative aspect through DATASUS/TABNET, using the variables, year of notification, year of diagnosis, year beginning of treatment, gender and closed situation involving healing, abandonment and death from tuberculosis and was. In this sense, the highest numbers of notifications occurred in the year 2018 and the lowest, in the year 2020. Regarding cure, treatment abandonment and death for tuberculosis in the indicated period, the highest percentages were: $79.35 \%$ in $2016,22.74 \%$ in 2020 and $10.83 \%$ in 2020 , respectively. Regarding gender, the highest percentage was male, being $74.27 \%$, which were associated with a lower cure rate, higher treatment abandonment rate and a higher rate of deaths from tuberculosis, when compared to females, present in $25.72 \%$ of total cases. Therefore, the results show a significant percentage of cases with male gender predominance related to the lower cure rate, higher dropout rate and higher death rate.
\end{abstract}

Keywords: Tuberculosis; Cure; Deaths; Male gender.

\begin{abstract}
Resumen
La tuberculosis es una enfermedad infecciosa causada por el bacilo Mycobacterium tuberculosis. Es una enfermedad prevenible y curable, pero a pesar de ello sigue siendo una grave amenaza para la salud pública mundial, siendo la segunda causa de muerte entre las enfermedades transmisibles. El estudio tiene como objetivo identificar la prevalencia y la adherencia al tratamiento en relación con los resultados curación, abandono y muerte por tuberculosis en el estado de Goiás en los años 2016 a 2020. Se trata de un estudio epidemiológico transversal de aspecto cuantitativo a través de DATASUS/TABNET, utilizando las variables, año de notificación, año de diagnóstico, año de inicio de tratamiento, género y situación cerrada que implica curación, abandono y muerte por tuberculosis y fue. En este sentido, el mayor número de notificaciones se produjo en el año 2018 y el menor, en el año 2020. En cuanto a la curación, el abandono del tratamiento y la muerte por tuberculosis en el periodo indicado, los mayores porcentajes fueron: $79,35 \%$ en $2016,22,74 \%$ en 2020 y 10,83\% en 2020, respectivamente. En cuanto al sexo, el mayor porcentaje fue el masculino, siendo el 74,27\%, que se asoció a una menor tasa de curación, mayor tasa de abandono del tratamiento y mayor tasa de muertes por tuberculosis, en comparación con el sexo femenino, presente en el $25,72 \%$ del total de casos. Por lo tanto, los resultados muestran un porcentaje significativo de casos con predominio en el género masculino relacionado con una menor tasa de curación, una mayor tasa de abandono y una mayor tasa de mortalidade.
\end{abstract}

Palabras clave: Tuberculosis; Curación; Muertes; Género masculino.

\title{
1. Introdução
}

A tuberculose (TB) é uma doença infecciosa e transmissível, causada pelo Mycobacterium tuberculosis, que afeta prioritariamente os pulmões, embora possa acometer outros sistemas. Em 1998, a Organização Mundial da Saúde (OMS) reconheceu a TB como uma epidemia global (Brasil, 2017). Apesar dos avanços notáveis no controle da TB, os quais promoveram uma redução de $2 \%$ na taxa de incidência da doença entre 2017 e 2018 a tuberculose ainda é um problema de saúde pública que continua sendo uma das doenças transmissíveis mais letais do mundo (Cazabon et al, 2017). Desse modo, estima-se que a doença matou 1,5 milhão de pessoas em 2014, contra 1,2 milhão de vítimas do HIV (WHO, 2015) e, foi uma das dez principais causas de morte no mundo em 2018.

Na classificação da OMS, o Brasil ocupa a $20^{\circ}$ posição mundial em incidência de TB e a $19^{\circ}$ posição no que se refere à coinfecção tuberculose/HIV (WHO, 2018; Penna, 2019). Em 2019, foram notificados no país 73.864 casos da doença, e 4.490 homens, mulheres e crianças morreram de tuberculose (Brasil, 2020). Sabe-se que a distribuição da TB no Brasil está relacionada às condições socioeconômicas da população, relação essa que implica diretamente na prevenção, no diagnóstico 
precoce e na adesão ao tratamento. Outros fatores podem influenciar a adesão ao tratamento: individuais (idade, comorbidades, nível de informação, atitudes e expectativas relativas à doença, ao tratamento e ao sistema de saúde); econômicos (acesso aos benefícios para suportar os custos associados ao tratamento); relacionados ao sistema de saúde (mecanismos que favoreçam a adesão, tais como acesso, motivação e supervisão do tratamento); e sociais (estigma e discriminação) (WHO, 2014; Rabah et aI, 2017).

A incidência da TB varia entre os países devido às condições socioeconômicas, estrutura política, organização dos serviços de saúde e implementação de programas nacionais de controle da tuberculose (PNCT) (Taylan et al, 2016) . O PNCT do Brasil preconiza ações de prevenção e vigilância epidemiológica precoce e tratamento adequado, além de estratégias operacionais para o Tratamento Diretamente Observado (TDO) e organização dos serviços de saúde tanto para os casos de TB sensível como para os de resistência (Brasil, 2011). No entanto, a TB ainda é uma doença de alta prevalência no país apesar de ser ofertado tratamento gratuito e dos programas de saúde para acompanhamento dos pacientes na Atenção Básica. Para melhorar essa realidade, são necessários estudos que busquem o porquê da lenta regressão da prevalência dos casos de TB no país, assim como fundamentem os motivos da não adesão ao tratamento, por parte dos pacientes, tendo em vista que é um tratamento que foca na não transmissão da infecção e na cura da pessoa doente. Este estudo visa identificar a prevalência e a adesão ao tratamento por pacientes acometidos pela TB no estado de Goiás, assim como desfechos relacionados ao tratamento.

\section{Metodologia}

Trata-se de um estudo epidemiológico transversal de aspecto quantitativo, que teve como delimitação territorial e temporal o estado de Goiás e o período compreendido entre 2016 a 2020. As informações sobre os casos confirmados de TB e a população utilizada para o cálculo de prevalência foram obtidos no site do Departamento de Informática do Sistema único de saúde (DATASUS). Ao acessar o site, foi selecionado na página a opção informações de saúde (TABNET), sendo após isso, redirecionado para outra página, onde foi escolhida a opção epidemiológicas e morbidade. Após isso, a doença selecionada foi TB, em abrangência geográfica: estado de Goiás, sendo as variáveis estudadas as seguintes: ano de notificação, ano de diagnóstico, ano início de tratamento, sexo e situação encerrada envolvendo a cura, abandono e óbito por TB. Para um melhor entendimento, foi realizada uma análise descritiva com abordagem quantitativa dos resultados da coleta de dados.

Os dados foram dispostos em forma de tabelas de distribuição número de casos, ano e o desfecho da doença na região centro-oeste do Brasil. Os valores calculados seguiram como base as fórmulas recomendadas para estudos epidemiológicos.

\section{Resultados}

A partir dos resultados obtidos no levantamento epidemiológico entre os anos de 2016 a 2020, verificou-se que o ano com maior número de casos foi o de 2018, assim como o ano de 2020 foi o com o menor número de casos notificados.

Em relação aos percentuais de cura, abandono e óbito por TB, no ano de 2016, os casos de cura foram de 79,35\%, os de abandono do tratamento de $16,47 \%$ e os de óbito por TB de $4,17 \%$. No ano de 2017 , os casos de cura foram de $77,11 \%$, os de abandono do tratamento de 19,07\% e os de óbito por TB de 3,81\%. Em 2018, os casos de cura foram de 78,06\%, os de abandono do TB de 18,34\% e os de óbito por TB de 3,58\%. Em 2019, os casos de cura foram de 79,19\%, os de abandono do tratamento de $16,90 \%$ e os de óbito por TB de 3,89\%. E em 2020, os casos de cura foram de $66,42 \%$, os de abandono do tratamento de 22,74\% e os de óbito por TB de 10,83\%. O total de casos foi de 4011. A figura apresenta um gráfico de casos de tuberculose no estado de Goiás e desfechos da doença: 
Gráfico 1. Casos de tuberculose no estado de Goiás e desfechos da doença.

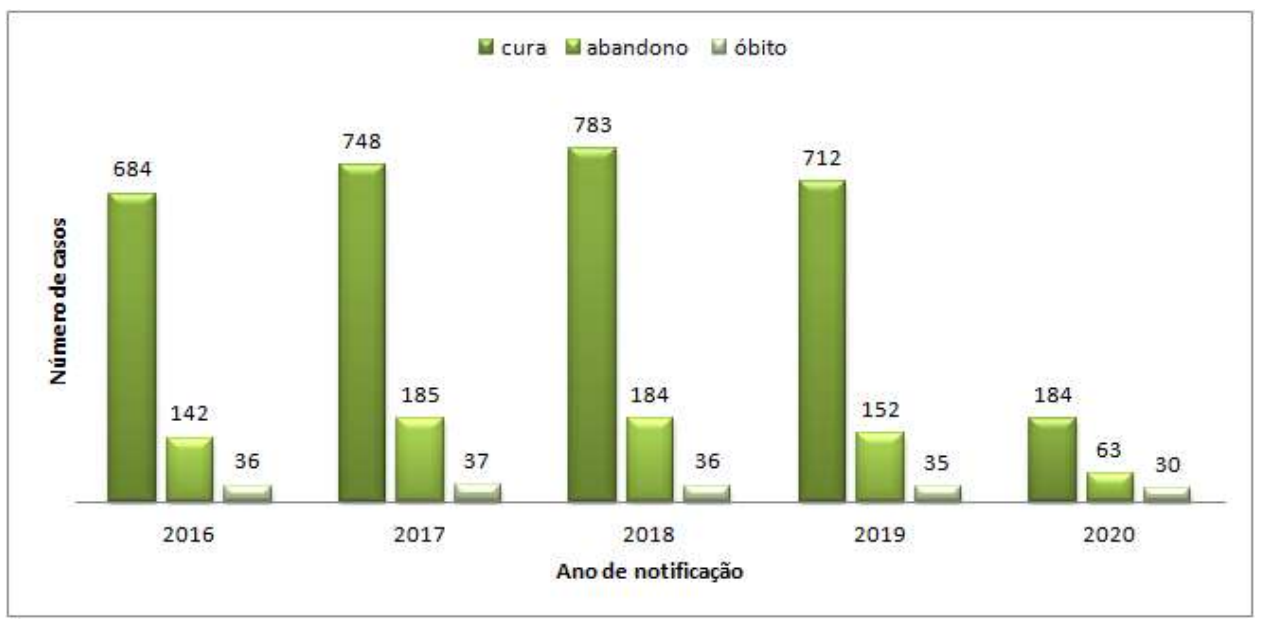

Fonte: Tabnet, DATASUS.

Relacionando-se os resultados obtidos de forma ampla aos resultados obtidos em relação ao sexo masculino, no ano de 2016, dos 614 casos do sexo masculino, os de cura foram referentes a 77,68\%, os de abandono do tratamento de $17,58 \%$ e os de óbito por TB de 4,72\%. No ano de 2017, dos 730 casos, os de cura foram referentes a 75,47\%, os de abandono do tratamento de 20,54\% e os de óbito por TB de 3,97\%. Em 2018, dos 756 casos, os de cura foram referentes a 75,92\%, os de abandono do tratamento de 20,37\% e os de óbito por TB de 3,70\%. No ano de 2019, dos 667 casos, os de cura foram referentes a 75,86\%, os de abandono do tratamento de $19,64 \%$ e os de óbito por TB de 4,49\%. Já no ano de 2020 , dos 212 casos, os de cura foram referentes a $63,20 \%$, os de abandono do tratamento de $25 \%$ e os de óbito por TB de $11,79 \%$. Somando 2979 casos. A figura representa um gráfico de casos de tuberculose em Goiás relacionados ao sexo masculino com desfechos da doença.

Gráfico 2. Casos de tuberculose no estado de Goiás relacionados ao sexo masculino e desfechos da doença.

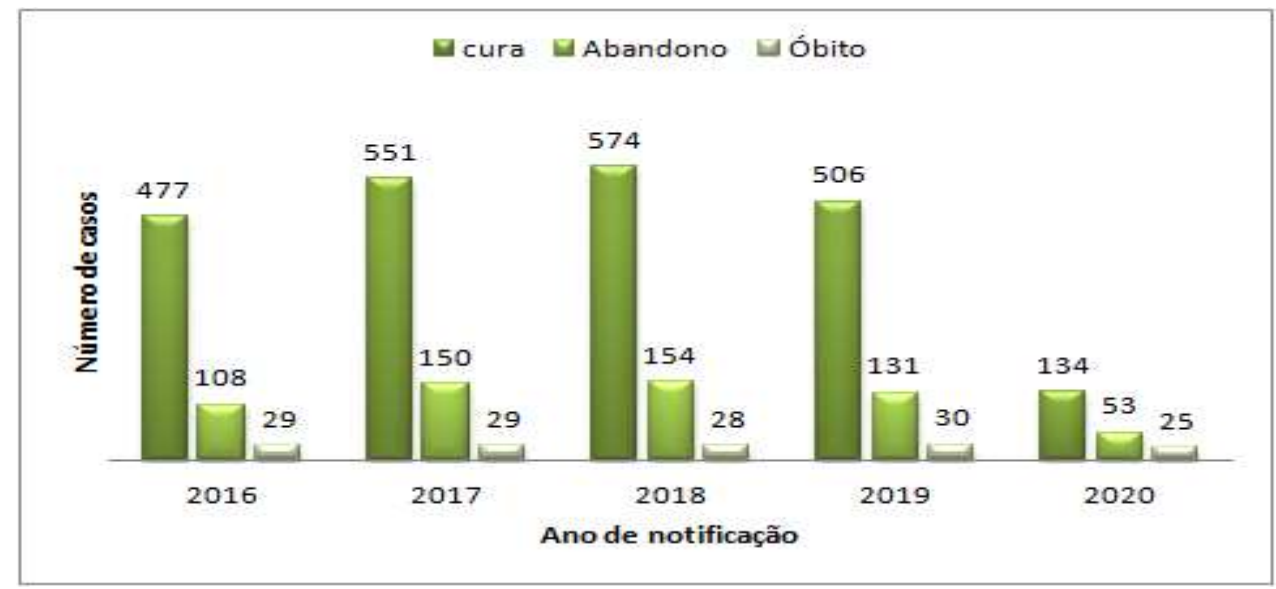

Fonte: Tabnet, DATASUS.

Em relação ao sexo feminino, no ano de 2016, dos 248 casos, os de cura foram referentes a 83,46\%, os de abandono do tratamento de $13,70 \%$ e os de óbito por TB de 2,82\%. No ano de 2017, dos 240 casos, os de cura foram referentes a $82,08 \%$, os de abandono do tratamento de $14,58 \%$ e os de óbito por TB de 3,33\%. No ano de 2018 , dos 246 casos, os de cura foram referentes a $84,55 \%$, os de abandono do tratamento de $12,19 \%$ e os de óbito por TB de 3,25\%. No ano de 2019 , dos 232 casos, os de cura foram referentes a $88,79 \%$, os de abandono do tratamento de $9,05 \%$ e os de óbito por TB de 2,15\%. Já no ano de 2020, dos 65 casos, os de cura foram referentes a 76,92\%, os de abandono do tratamento de 15,38\% e os de óbito por TB de 
7,69\%. Somando 1031 casos. A figura representa um gráfico de tuberculose em Goiás relacionados ao sexo feminino e aos desfechos da doença:

Gráfico 3. Casos de tuberculose no estado de Goiás relacionados ao sexo feminino e desfechos da doença.

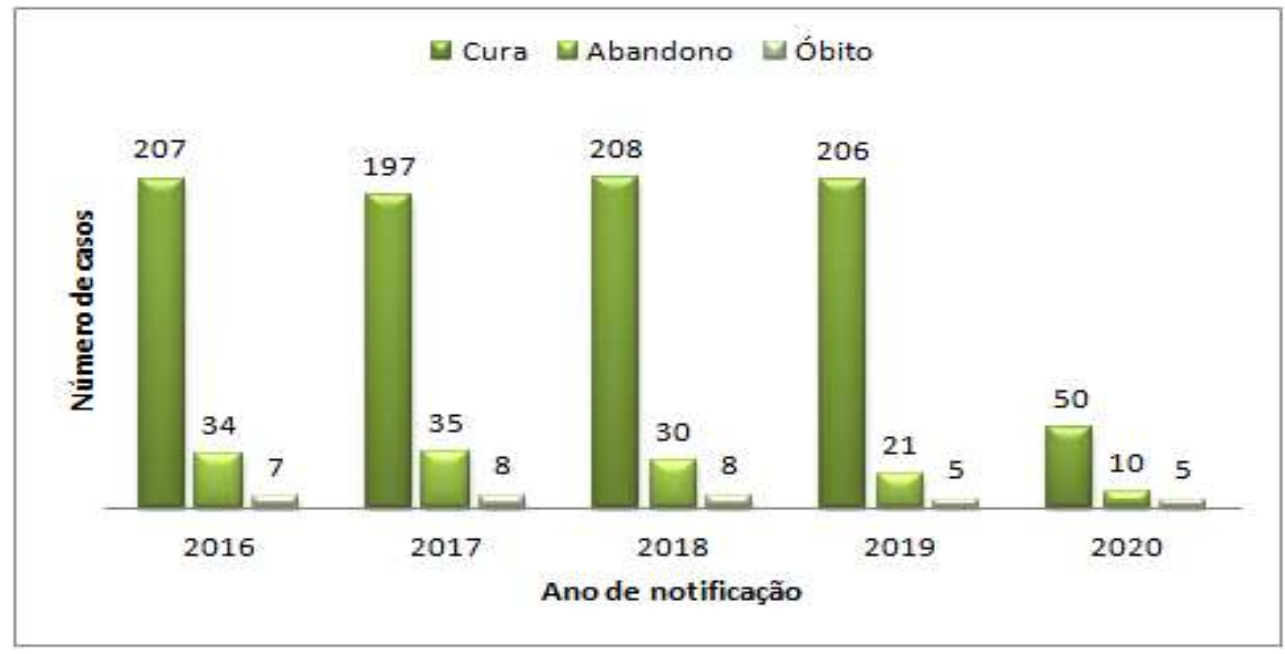

Fonte: Tabnet, DATASUS.

\section{Discussão}

A partir dos resultados obtidos, foi possível observar que, dos casos totais de TB no período analisado, a maior porcentagem foi a do sexo masculino, totalizando $74,27 \%$, que foram associados a uma menor taxa de cura, maior taxa de abandono do tratamento e a uma maior taxa de óbitos por TB, quando comparados com o sexo feminino, presente em $25,72 \%$ dos casos totais.

Esse fato traz consigo características da epidemiologia da doença em que a TB é mais prevalente em homens, assim como é uma das principais causas de morte entre adultos (Belo, 2010).

Além disso, analisando-se o período pesquisado, evidencia-se que, o ano de 2020 obteve uma taxa inferior a todos os outros quatro anos anteriores, na totalidade de casos, consolidando o fato de que, com a pandemia da COVID-19, houve uma subnotificação de casos de TB, assim como a diminuição da adesão ao tratamento, diminuindo-se consequentemente, os casos de cura e aumentando os casos de abandono e óbito por TB percentualmente. Dados esses que vão ao encontro com resultados obtidos no boletim epidemiológico TB 2021, que destaca que em 2020, em momento de pandemia pela COVID-19, uma queda acentuada da incidência em comparação com o ano anterior. Assim como observou-se uma queda de $16 \%$ na notificação de casos novos de TB em comparação com 2019, sendo que essa redução foi mais pronunciada a partir do mês de abril. Vale ressaltar que, o diagnóstico da TB durante a pandemia requer que os profissionais de saúde tenham uma alta suspeição clínica, já que, sintomas como, febre e sintomas respiratórios, são características das duas doenças, assim como as duas podem se apresentar de forma simultânea. Exames como tomografia computadorizada de tórax, facilita a detecção de infecção tuberculosa preexistente não diagnosticada (Silva, 2021; Mello et al, 2021).

Apesar dos resultados obtidos mostrarem um menor número de casos em 2020, há uma contraposição em relação a esses dados. Isso porque, apesar dessa menor taxa, houve também uma menor taxa de cura e uma maior taxa de abandono e óbito por TB. Assim, estima-se que 1,4 milhão de pessoas a menos receberam tratamento para TB em 2020 em relação ao ano anterior, 2019, de acordo com dados preliminares compilados pela OMS em mais de 80 países - uma redução de $21 \% \mathrm{em}$ relação a 2019 (Fiocruz).

Em relação ao abandono do tratamento, as taxas de abandono do sexo masculino foram maiores que as do sexo 
feminino. O abandono de tratamento está relacionado a alguns fatores, principalmente devido aos sociodemográficos, ocorrendo principalmente em homens com menor escolaridade, assim como, relaciona-se também ao consumo de drogas como o álcool. Além disso, a associação com outras doenças crônicas, mais especificamente a infecção pelo HIV, está relacionada ao abandono de tratamento da TB (Chirinos, Meirelles, 2011)

A associação entre o tratamento medicamentoso da tuberculose e o consumo de álcool aumenta a chance de intolerância à medicação, o que pode também ser considerado como uma das causas de abandono. Dentre outras causas estão o fato do paciente não suportar os efeitos colaterais do tratamento devido à falta de condições econômicas de se alimentarem de forma correta e também à situação da população carcerária que, em muitos casos, tem o acesso ao medicamento dificultado, assim como o pensar do paciente em relação a estar curado, pelo fato dos sintomas terem tido uma regressão no início do tratamento, chegando-se assim na conclusão de não necessidade de continuar o tratamento que deveria ser de seis meses (Couto, et al 2014).

\section{Conclusão}

Diante dos resultados expostos do levantamento epidemiológico da TB no estado de Goiás entre os anos de 2016 a 2020, que demonstram uma porcentagem significante dos casos com predominância no gênero masculino relacionados a menor taxa de cura, maior taxa de abandono ao tratamento e maior taxa de óbitos, é evidente a importância desse estudo para a área científica e saúde pública, uma vez que possibilita identificar o panorama da tuberculose em Goiás. Essa prevalência dos casos de tuberculose em adultos do sexo masculino provavelmente acontece em virtude dos hábitos comportamentais e sociodemográficos que deixam este grupo mais vulnerável ao consumo de drogas como o álcool. No estudo foi possível observar que o abandono ao tratamento pode estar associado a casos diagnosticados com coinfecção TB/HIV. Além disso, o levantamento dos dados mostrou uma queda dos números de casos no ano de 2020, o que demonstra uma subnotificação dos casos de TB em decorrência do COVID-19. É de grande relevância também as questões relacionadas aos serviços de saúde, onde a ausência de busca ativa para o diagnóstico de novos casos, a deficiência do vínculo com os profissionais de saúde, o despreparo, e a desorganização da equipe contribuem para a desistência dos doentes ao tratamento.

Por fim, estes resultados demonstram que é necessário o fortalecimento dos programas de promoção e de tratamento da saúde, a fim de sensibilizar a população, para a redução do abandono ao tratamento e, consequentemente, seja alcançado o controle da prevalência de TB no estado de Goiás. No mais, é importante que sejam feitos estudos pós pandemia com intuito de demonstrar se haverá aumento da incidência de TB com intuito de atestar as subnotificações de casos no período pandêmico.

\section{Referências}

Belo, M. T. C. T., Luiz, R. R., Hanson, C., Selig, L., Teixeira, E. G., Chalfoun, T., \& Trajman, A. (2010). Tuberculose e gênero em um município prioritário no estado do Rio de Janeiro. Jornal Brasileiro de Pneumologia, 36, 621-625.

Brasil. (2017). Ministério da Saúde. Sala de Apoio à Gestão Estratégica (SAGE). Brasília: Situação de saúde e indicadores de morbidade da tuberculose. SAGE; c2017.

Brasil (2021). Doenças de Condições Crônicas e Infecções Sexualmente Transmissíveis.

Brasil (2020). Ministério da Saúde. Secretaria de Vigilância em Saúde. Brasília: Boletim Epidemiológico.

Cazabon, D., Alsdurf, H., Satyanarayana, S., Nathavitharana, R., Subbaraman, R., Daftary, A., \& Pai, M. (2017). Qualidade da atenção à tuberculose em países com alta carga: a necessidade urgente de abordar as lacunas na cascata de atenção. International Journal of Infectious Disease, $56,111-116$.

Couto, D. S. D., Carvalho, R. N., Azevedo, E. B. D., Moraes, M. N. D., Pinheiro, P. G. O. D., \& Faustino, E. B. (2014). Fatores determinantes para o abandono do tratamento da tuberculose: representações dos usuários de um hospital público. Saúde em Debate, 38, $572-581$.

Chirinos, N. E. C., \& Meirelles, B. H. S. (2011). Fatores associados ao abandono do tratamento da tuberculose: uma revisão integrativa. Texto \& ContextoEnfermagem, 20, 599-606. 
Research, Society and Development, v. 10, n. 11, e556101119869, 2021

(CC BY 4.0) | ISSN 2525-3409 | DOI: http://dx.doi.org/10.33448/rsd-v10i11.19869

DATASUS. Casos de Tuberculose-Desde 2001 (SINAN).

Fiocruz. (2019). Pandemia limita ações para a erradicação da tuberculose.

Mello, F. C. D. Q., D’Ambrosio, L., Centis, R., Dalcolmo, M. P., \& Migliori, G. B. (2021). Tuberculose e COVID-19, o novo dueto maldito: quais as diferenças entre Brasil e Europa? Jornal Brasileiro de Pneumologia, 47.

Ministério da Saúde (BR), \& Secretaria de Vigilância em Saúde. (2019). Manual de recomendações para o controle da tuberculose no Brasil [Internet].

Penna, G. (2011). Manual de Recomendações para o Controle da Tuberculose no Brasil.

Rabahi, M. F., Silva, J. L. R. D., Ferreira, A. C. G., Tannus-Silva, D. G. S., \& Conde, M. B. (2017). Tratamento da tuberculose. Jornal brasileiro de pneumologia, 43, 472-486.

Secretaria de Estado da Saúde. (2015). Superintendência de Vigilância em Saúde EM SA Ú D E Gerência de Vigilância Epidemiológica Situação Epidemiológica e Operacional da Tuberculose no Estado de Goiás. - Programa Estadual de Controle da Tuberculose/CDCT/GVE/SUVUSA/SES-GO

Silva, D. R., Mello, F. C. D. Q., \& Migliori, G. B. (2021). Série Tuberculose 2021.Silva, D. R.

Taylan, M., Demir, M., Y1lmaz, S., Kaya, H., Sen, H. S., Oruc, M., \& Sezgi, C. (2016). Effect of human development index parameters on tuberculosis incidence in Turkish provinces. The Journal of Infection in Developing Countries, 10(11), 1183-1190.

TABNET. Casos confirmados notificados no sistema de informação de agravos de notificação-Goiás- BRASIL.

Taylan, M., Demir, M., Yılmaz, S., Kaya, H., Sen, H. S., Oruc, M. \& Sezgi, C. (2016). Effect of human development index parameters on tuberculosis incidence in Turkish provinces. The Journal of Infection in Developing Countries, 10(11), 1183-1190.

World Health Organization. (2014). Companion handbook to the WHO guidelines for the programmatic management of drug-resistant tuberculosis. World Health Organization.

World Health Organization. (2015). Global tuberculosis report 2015. World Health Organization. 